\title{
Phased implementation of screening for cryptococcal disease in South Africa
}

Cryptococcus neoformans is the most common cause of laboratoryconfirmed meningitis in South Africa. ${ }^{1}$ Despite the increased coverage of antiretroviral treatment (ART), the country's incidence of cryptococcal meningitis remains high, and in routine care settings, the disease has a case-fatality ratio of $>50 \%$ at 12 weeks post-diagnosis. ${ }^{2-4}$ Screening and pre-emptive antifungal treatment is desirable to prevent the development of cryptococcal meningitis and associated deaths. ${ }^{5-7}$

Implementing a high-throughput screening programme has been simplified by the development of a sensitive and specific lateral flow assay (LFA) (Immuno-Mycologics, Norman, OK, USA) designed to rapidly detect cryptococcal antigen $(\mathrm{CrAg})$ in several body fluids. ${ }^{8,9}$

In its Rapid Advice guidelines, the World Health Organization (WHO) indicates that, before initiating ART in populations with a high prevalence of cryptococcal antigenaemia, routine screening for cryptococcal disease may be considered among ART-naïve adults who have a CD4+ T-lymphocyte count $<100$ cells $/ \mu \mathrm{l} .{ }^{10}$ In two South African ART cohorts, the prevalence of incident antigenaemia among patients with a CD4+ T-lymphocyte count $<100$ cells/ $\mu$ l was $4 \%$ and $7 \%$ respectively. ${ }^{11,12}$ This is greater than the breakpoint above which screening was found to be potentially cost-saving in a Ugandan study. ${ }^{13,14}$

To reduce disability and deaths associated with HIV infection, it has been suggested that laboratory-based screening and preemptive antifungal treatment of cryptococcal disease be routinely implemented as part of the South African National Strategic Plan for HIV, STIs and TB, 2012-2016. ${ }^{15}$ This initiative also complements government's efforts to prioritise primary health care (PHC), with a shift towards early detection of cryptococcal disease at PHC clinics rather than delayed diagnosis of cryptococcal meningitis at hospital level. The National Department of Health has recommended phasing in cryptococcal screening to the provincial departments (Dr Yogan Pillay, personal communication).

\section{Phased laboratory-based implementation}

In the first phase of the programme, lab-based screening will begin at almost 500 health facilities in Gauteng and the Free State, served by three National Health Laboratory Service (NHLS) laboratories performing CD4+ T-lymphocyte testing. In a lab-based strategy, NHLS laboratories will reflexively test the remnant specimen of any blood sample with CD4+ T-lymphocyte count $<100$ cells/ $\mu$ l for $\mathrm{CrAg}$ using the LFA. Results will be reported to the clinician, together with CD4+ T-lymphocyte count results, using existing paper and electronic methods.

A phased approach will establish the feasibility and appropriate workflow in these labs. This strategy was selected over point-of-care (POC) testing for several reasons.

- First, over 400000 specimens submitted for CD4+ T-lymphocyte testing to 62 NHLS laboratories nationwide in 2011 had a CD4+ T-lymphocyte count $<100$ cells $/ \mu$ l - i.e. $11 \%$ of all submitted specimens (Professor Wendy Stevens, personal communication). In this context, rapidly scaling up implementation beyond the first phase is more feasible with a lab-driven strategy.

- Second, with reflex testing, additional venepuncture would be unnecessary and healthcare providers would not need to remember to order the test on patients with a CD4+ T-lymphocyte count $<100$ cells $/ \mu$. This simplifies the screenand-treat algorithm.
- Third, unless a POC CD4+ T-lymphocyte test is also offered at a health facility, a POC LFA would only be done at a followup visit when the laboratory CD4+ T-lymphocyte test result is available. This offers no advantage in terms of turn-around time. POC CD4+ T-lymphocyte testing is currently available at only a few health facilities in South Africa (Professor Wendy Stevens, personal communication). In the future, if the LFA is validated on whole blood or urine, it could be used as a POC test.

\section{Training and clinical management}

Healthcare and lab workers must be trained as the screening programme is implemented. Training healthcare workers is particularly important because the lab-based screening is not provider-initiated. Training will consist of a half-day, module-based workshop focused on case studies and followed by in-service training and mentoring.

There is currently no standard treatment practice for patients with no symptoms and signs of meningitis who screen positive for $\mathrm{CrAg}$. Current evidence has been used to inform the clinical treatment algorithm for this programme (Fig. 1). ${ }^{10,16,17}$ For patients testing CrAg-positive, clinicians will first evaluate patients for symptoms and signs of meningitis (including headache for longer than 24 hours, fever, confusion or reduced level of consciousness, blurred vision, or neck stiffness). The clinician will also evaluate patients for any special situations (previous history of cryptococcal meningitis, pregnancy, liver disease, nevirapine use and age $<18$ years).

Patients with symptoms or signs of meningitis will be started immediately on oral fluconazole (400 mg once daily) and have a lumbar puncture performed, or be transferred urgently to a facility where a lumbar puncture can be performed, to exclude cryptococcal meningitis through cerebrospinal fluid (CSF) analysis. Patients with a positive lumbar puncture for cryptococcal meningitis will be treated according to South African cryptococcal disease guidelines (amphotericin B-based induction treatment for 2 weeks, followed by fluconazole $400 \mathrm{mg}$ daily for 8 weeks, followed by secondary prophylaxis, $200 \mathrm{mg}$ until the CD4+ T-lymphocyte count $>200$ cells/ $\mu \mathrm{l}$ for six months on ART) ${ }^{17,18}$ For patients without symptoms of meningitis or symptomatic patients with a negative lumbar puncture, treatment with fluconazole ( $400 \mathrm{mg}$ for 8 weeks and then $200 \mathrm{mg}$ until CD4+ T-lymphocyte count $>200$ cells/ $\mu$ f for 6 months on ART) is recommended.

Note that the WHO Rapid Advice guidelines recommend an $800 \mathrm{mg}$ daily initial dose of fluconazole. The relatively low dose of $400 \mathrm{mg}$ daily was only selected because fluconazole is not currently available at the higher dose at PHC facilities. The algorithm may be revised if evidence suggests that a higher dose is more efficacious for asymptomatic antigenaemia or reduces duration of treatment. ${ }^{10,18}$ Patients without symptoms of meningitis may also be offered a lumbar puncture if it can be performed at the health facility where the patient was diagnosed or if they can be rapidly referred to a facility that offers it. If lumbar puncture is positive for cryptococcal meningitis in asymptomatic patients, the patient should be treated as described above. For CrAg-positive patients, ART will be started two weeks after antifungal therapy is initiated. ${ }^{10}$ However, ART initiation may need to be delayed to 4 weeks after diagnosis among patients with confirmed cryptococcal meningitis (Professor Graeme Meintjes, personal communication). 


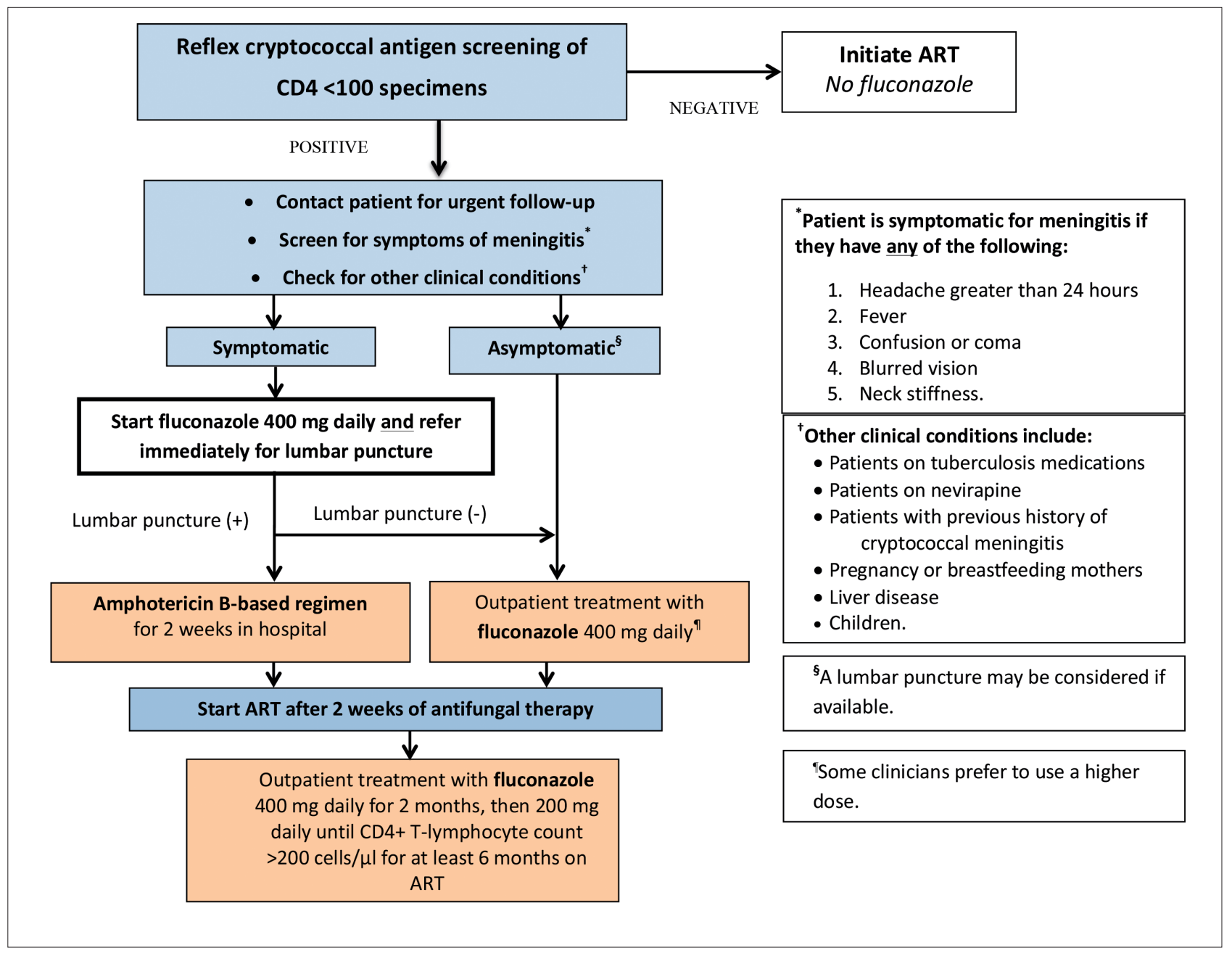

Fig. 1. Clinical treatment algorithm for the South African cryptococcal screening programme.

Recommendations (Note: This algorithm may be modified as new evidence is published.)

- Patients on TB medications. Anti-TB drugs (including INH) and fluconazole can be started at the same time. Because both fluconazole and TB medications are potentially hepatotoxic, these patients should preferably be started on an efavirenz-based ART regimen. Patients should be monitored closely for signs of liver damage including right upper quadrant abdominal pain, nausea/vomiting, or jaundice. If there are signs of toxicity, then liver function tests should be ordered. - Patients on nevirapine. For patients starting ART, an efavirenz-based regimen is preferable to nevirapine, since nevirapine might increase the risk of liver damage. If patient is already on nevirapine, then the patient should be monitored closely for signs of liver damage including right upper quadrant abdominal pain, nausea/vomiting, or jaundice. If there are signs of toxicity, then liver function tests should be ordered.

- Patients with previous history of cryptococcal meningitis. Patients with a previous history do not need to be routinely screened. However, patients who have new symptoms of meningitis will need to be evaluated for relapse disease and/or immune reconstitution inflammatory syndrome (IRIS). If the patient does not have new symptoms, the healthcare provider should ensure that the patient has received or is receiving adequate maintenance therapy (fluconazole

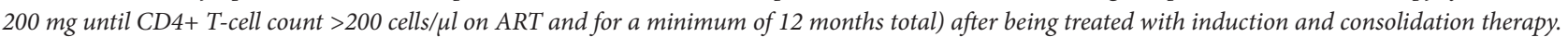
- Pregnancy or breastfeeding mothers. Because fluconazole can be harmful to a fetus, all women of childbearing age should have a pregnancy test. The risks, benefits and alternatives to fluconazole treatment should be discussed with the patient. Consultation with a physician experienced in the care of HIV-infected patients is recommended. Breastfeeding mothers also require consultation with an experienced physician as fluconazole can be transmitted through breast milk to the infant. Women of childbearing age who are not yet pregnant and are starting fluconazole treatment should be advised to avoid becoming pregnant during treatment.

- Liver disease. Patients with history of liver disease (cirrhosis, hepatitis, etc.), jaundice, or abnormal liver enzyme tests (more than twice upper limit of normal) require careful monitoring because fluconazole may cause liver damage. Consultation with a physician experienced in the care of HIV/AIDS patients is recommended.

- Children. Screening is not recommended for children as cryptococcal meningitis is less common in this group. All children who are CrAg-positive should be referred for lumbar puncture (LP). Children with a positive LP should be managed according to the national guidelines: amphotericin $B 1 \mathrm{mg} / \mathrm{kg} /$ dose IV for at least 2 weeks, followed by fluconazole 12 - $15 \mathrm{mg} / \mathrm{kg}$ daily for 8 weeks, followed by fluconazole 6 - $10 \mathrm{mg} / \mathrm{kg}$ daily for life or until the CD4\% >25\% (if 2 - 6 years old) or CD4+ T-lymphocyte count $>200$ cells/ul (if >6 years old); children aged $<2$ years should be continued on treatment for life. Children with a negative LP should be treated with a weight-based fluconazole regimen of $12-15 \mathrm{mg} / \mathrm{kg}$ daily for 8 weeks, followed by $6-10 \mathrm{mg} / \mathrm{kg}$ daily until the CD $4 \%>25 \%$ (if $\leq 6$ years old) or CD4+ T-lymphocyte count $>200$ cells/ $\mu$ l (if $>6$ years old). Daily fluconazole dose for children should never exceed 400 mg.

- ART initiation. ART may need to be delayed to 4 weeks after diangnosis in patients with confirmed cryptococcal meningitis. 
A data safety monitoring committee, consisting of South African clinicians who are experts in the field of HIV management, will convene to regularly review the treatment algorithm, any reported adverse events and programme indicator data.

\section{Monitoring and evaluation}

The primary goal of cryptococcal screening is to reduce morbidity and mortality associated with cryptococcal meningitis in South Africa. Initially, screening may increase the number of incident cases of cryptococcal disease, and improved detection may increase the number of cases of meningitis. However, these are expected to plateau as national screening is implemented and coverage is expanded.

Incidence of cryptococcal disease and the in-hospital casefatality ratio will be monitored by the national Group for Enteric, Respiratory, and Meningeal Surveillance-South Africa (GERMSSA) surveillance programme. ${ }^{4}$ The programme's impact on overall mortality reduction will be assessed by comparing 6- and 12-month mortality among HIV-infected patients who are newly initiated on ART before and after implementing cryptococcal screening at selected participating clinics. This process will be facilitated by the routine electronic capture of ART programme data, including outcome data, on TIER.net (Dr Meg Osler, personal communication). Laboratory and clinical indicator data, collected as part of the screening programme, will be used to perform a cost-effectiveness analysis, including costs of screening per patient and outcome stratified by treatment. The aim is to demonstrate that screening and treating patients with early cryptococcal disease (asymptomatic antigenaemia and early cryptococcal meningitis) is more cost-effective than treating patients with cryptococcal meningitis who are diagnosed only when symptomatic.

\section{Operational challenges}

Several operational challenges must be addressed as screening is implemented. First, cryptococcal screening must be integrated into routine management algorithms for patients who require rapid ART initiation and may also have TB. Second, for the programme to work, patients found to have cryptococcal antigenaemia must be immediately traced, assessed and initiated on antifungal treatment before they develop meningitis or die. Laboratory reporting and clinic tracing systems need to be enhanced. Third, supply, procurement and distribution of antifungal drugs to clinic level must be adequate and consistent, and nurses who identify patients with antigenaemia need to be able to initiate antifungal treatment. The broader issues of data collection for programme monitoring and evaluation, integration of vertical programmes, patient follow-up and drug supply are not specific to the cryptococcal screening programme.

The National Department of Health has recommended phasing in screening to provinces, and the Western Cape provincial government has already updated its ART programme guidelines to include cryptococcal screening (Professor Graeme Meintjes, personal communication). Screening should be prioritised across the country because the South African cryptococcal screening programme can significantly mitigate the burden and death rate associated with this common and deadly fungal opportunistic infection.

Members of the South African Cryptococcal Screening Initiative Group: National Department of Health: Yogan Pillay, Thobile Mbengashe. Gauteng Department of Health: Zukiswa Pinini, Lucky Hlatshwayo, Nobantu Mpela. Free State Department of Health: Yolisa Tsibolane. Right to Care: David Spencer, Inge Harlen, Barbara Franken, Shabir Banoo, Pappie Majuba, Ian Sanne. Wits Reproductive and HIV Research Institute: W D Francois Venter, Ambereen Jaffer, Bongiwe
Zondo, Judith Mwansa, Andrew Black, Thilligie Pillay, Mamotho Khotseng, Vivian Black. Aurum: Dave Clark. Health Systems Trust: Waasila Jassat, Richard Cooke, Petro Rousseau. Anova: James McIntyre, Kevin Rebe, Helen Struthers. BroadReach: Mpuma Kamanga, Mapule Khanye, Madaline Feinberg, Mark Paterson. Technical advisors: Tom Chiller, Monika Roy (CDC Atlanta); Samuel Oladoyinbo, Adeboye Adelakan (CDC South Africa); Thapelo Maotoe (USAID South Africa). Expert clinicians: Jeffrey Klausner, Tom Harrison, Joseph Jarvis, Nicky Longley, Robin Wood, Stephen Lawn, Linda-Gail Bekker, Gary Maartens, Francesca Conradie. Data Safety and Monitoring Committee: Graeme Meintjes, Yunus Moosa, Halima Dawood, Kerrigan McCarthy, Alan Karstaedt. National Health Laboratory Service: Wendy Stevens, Lindi Coetzee, Debbie Glencross, Denise Lawrie, Naseem Cassim, Floyd Olsen. National Institute for Communicable Diseases/NHLS: Verushka Chetty, Nelesh Govender.

Disclaimer. The findings and conclusions of this article are those of the authors and do not necessarily represent the official position of the Centers for Disease Control and Prevention. The use of product names in this manuscript does not imply their endorsement by the USA Department of Health and Human Services.

\section{The South African Cryptococcal Screening Initiative Group:}

\section{N P Govender}

V Chetty

National Institute for Communicable Diseases, a division of the National Health Laboratory Service - Centre for Opportunistic, Tropical and Hospital Infections, Johannesburg

\section{N P Govender}

Faculty of Health Sciences, University of the Witwatersrand, Johannesburg

\section{Roy}

T Chiller

Mycotic Diseases Branch, Centers for Disease Control and Prevention, Atlanta, USA

\section{S Oladoyinbo}

Care and Treatment Branch, Centers for Disease Control and Prevention, Pretoria

\section{T Maotoe}

Care and Treatment Branch, United States Agency for International Development, Pretoria

\section{W S Stevens}

National Priority Programmes Unit, National Health Laboratory Service and Department of Molecular Medicine and Haematology, University of the Witwatersrand, Johannesburg

\section{Z Pinini}

HIV/AIDS, STIs and TB Cluster, Gauteng Department of Health, Johannesburg, South Africa

\section{Spencer}

Right to Care, Johannesburg

\section{W D F Venter}

Wits Reproductive Health and HIV Institute and Department of Medicine, University of the Witwatersrand, Johannesburg 


\section{EDITORIAL}

W Jassat

Health Systems Trust, Midrand

D Cameron

Foundation for Professional Development, Pretoria

\section{G Meintjes}

Institute of Infectious Diseases and Molecular Medicine and Department of Medicine, University of Cape Town; and Department of Medicine, Imperial College London

\section{T Mbengashe \\ Y Pillay}

National Department of Health, Pretoria

Corresponding author: N P Govender (neleshg@nicd.ac.za)

1. Jarvis JN, Meintjes G, Williams A, Brown Y, Crede T, Harrison TS. Adult meningitis in a setting of high HIV and TB prevalence: findings from 4961 suspected cases. BMC Infect Dis 2010;10:67. [http:// dx.doi.org/10.1186/1471-2334-10-67.

2. Jarvis JN, Boulle A, Loyse A, et al. High ongoing burden of cryptococcal disease in Africa despite antiretroviral roll out. AIDS 2009;23(9):1182-1183. [http://dx.doi.org/10.1097/ QAD.0b013e32832be0fc]

3. Park BJ, Shetty S, Ahlquist A, et al. Long-term follow-up and survival of antiretroviral-naive patients with cryptococcal meningitis in the pre-antiretroviral therapy era, Gauteng Province, South Africa. Int J STD AIDS 2011;22(4):199-203. [http://dx.doi.org/10.1258/ijsa.2010.010235]

4. National Institute for Communicable Diseases. GERMS-SA Annual Report 2011, Johannesburg: National Institute for Communicable Diseases, 2012. http://www.nicd.ac.za (accessed 5 September 2012).

5. Jarvis JN, Lawn SD, Wood R, Harrison TS. Cryptococcal antigen screening for patients initiating antiretroviral therapy: time for action. Clin Infect Dis 2010;51(12):1463-1465. [http://dx.doi org/10.1086/657405]
6. Jarvis IN, Harrison TS, Govender N, et al. Routine cryptococcal antigen screening for HIV-infected patients with low CD4+ T-lymphocyte counts - time to implement in South Africa? S Afr Med (4):232-234.

7. Klausner JD, Govender N, Oladoyinbo S, Roy M, Chiller T. Preventing AIDS deaths: cryptococcal antigen screening and treatment. Lancet Infect Dis 2012;12(6):431-432.

8. Jarvis IN, Percival A, Bauman S, et al. Evaluation of a novel point of care cryptococcal antigen (CRAG) test on serum, plasma and urine from patients with HIV-associated cryptococcal meningitis. Clin Infect Dis 2011;53(10):1019-1023. [http://dx.doi.org/10.1093/cid/cir613]

9. Rajasingham R, Meya DB, Boulware DR. Integrating cryptococcal antigen screening and pre-emptive treatment into routine HIV care. J Acquir Immune Defic Syndr 2012;59(5):e85-91.

10. World Health Organization. Rapid Advice: Diagnosis, Prevention and Management of Cryptococca Disease in HIV-infected Adults, Adolescents and Children. Geneva: World Health Organization, 2011 www.who.int/hiv/pub/cryptococcal_disease2011/en/ (accessed 5 September 2012).

11. Jarvis J, Lawn S, Vogt M, Bangani N, Wood R, Harrison TS. Screening for cryptococcal antigenaemia among patients in an antiretroviral treatment program in South Africa. Clin Infect Dis 2009;48(7):856862. [http://dx.doi.org/10.1086/597262]

12. Roy M, Karstaedt AS, Mendes J, et al. Evaluation of a cryptococcal antigen screening program for HIV-infected persons initiating anti-retroviral therapy in South Africa (Abstract TUAB0503). In Abstracts: 16th International Conference on AIDS and STIs in Africa, Addis Ababa, Ethiopia, 2011:4-8.

13. Meya DB, Manabe YC, Castelnuovo B, et al. Cost-effectiveness of serum cryptococcal antigen screening to prevent deaths among HIV-infected persons with a CD4+ cell count $\leq 100 \mathrm{cells} / \mu \mathrm{L}$ who start HIV therapy in resource-limited settings. Clin Infect Dis 2010;51(4):448-455. [http://dx.do org/10.1086/655143]

14. Rajasingham R, Boulware DR. Reconsidering cryptococcal antigen screening in the U.S. amon persons with $\mathrm{CD} 4<100$ cells/ $/ \mathrm{L}$. Clin Infect Dis 2012 (in press).

5. South African National AIDS Council. National Strategic Plan on HIV, STIs and TB, 2012-2016. Pretoria: South African National AIDS Council, 2012. http://www.sanac.org.za/index.php/resources/ national-strategic-plan (accessed 5 September 2012).

16. Jarvis JN, Govender NP, Chiller T, et al. Cryptococcal antigen screening and pre-emptive therapy in patients initiating antiretroviral therapy in resource-limited settings: a proposed algorithm for clinical implementation. J Int Assoc Physicians AIDS Care 2012 (in press).

17. McCarthy K, Meintjes G, Arthington-Skaggs B, et al. Guidelines for the diagnosis, management and prevention of cryptococcal meningitis and disseminated cryptococcosis in HIV-infected patients. S Afr J HIV Med 2007;Spring Edition:25-35.

18. National Department of Health. Standard Treatment Guidelines and Essential Drugs List for South Africa: Primary Health Care. Pretoria: National Department of Health 2008. http://www.kznhealth. gov.za/edlphc2008.pdf (accessed 5 September 2012).

S Afr Med J 2012;102(12):914-917. DOI:10.7196/SAMJ.6228 